\title{
Successful treatment of pulmonary mucormycosis, a rare pulmonary fungal infection, in a patient with diabetes mellitus
}

Yukihiro Zaizen, MD, PhD, and Tatsuya Ohtsu, MD, Beppu, Japan

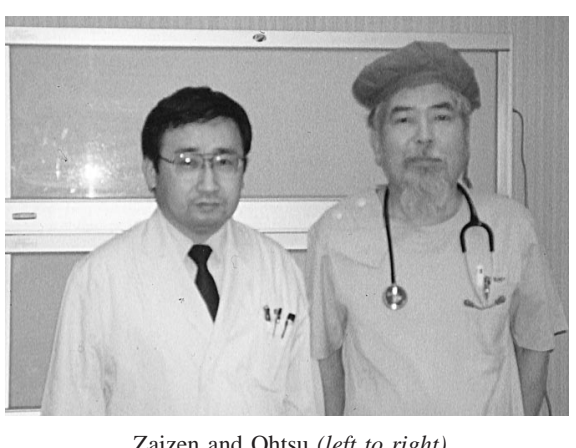

A lthough commonly found in our environment, some fungi are potentially pathogenic and may cause diseases resembling pneumonia. These infections occur mainly in human beings with various predisposing conditions. Pulmonary mucormycosis is uncommon but is an acute and life-threatening disease. The disease can progress from the acute phase to stages characterized by exac-

From the Department of Respiratory Disease, National Nishibeppu Hospital, Beppu, Japan.

Received for publication Feb 12, 2002; accepted for publication March 27, 2002.

Address for reprints: Yukihiro Zaizen, MD, PhD, Department of Respiratory Disease, National Nishibeppu Hospital, Tsurumi 4548, Beppu, Oita 874-0838, Japan (E-mail: zaizeny@ wbeppu.hosp.go.jp).

J Thorac Cardiovasc Surg 2002;124:838-40

Copyright $\odot 2002$ by The American Association for Thoracic Surgery

$0022-5223 / 2002 \$ 35.00+0 \quad \mathbf{1 2 / 5 4} / \mathbf{1 2 5 2 8 3}$

doi: $10.1067 / \mathrm{mtc} .2002 .125283$ erbation and finally to end-stage septicemia. ${ }^{1-3}$ A diagnosis of mucormycosis can usually be made at autopsy.

\section{Clinical Summary}

A 64-year-old man was admitted to hospital with the complaints of nausea, chest pain, dyspnea, and general malaise. He gave a history of heavy alcohol intake (4 U/d), excessive smoking (Brinkman index 900), and poorly controlled diabetes mellitus.

Two weeks before admission, he had become nauseated and started to have paroxysmal nocturnal dyspnea and night sweats. $\mathrm{He}$ attended a private clinic, but the dyspnea and nausea became increasingly troublesome, despite treatment with antibiotics and antipyretics. Chest radiography revealed an area of consolidation in the right lung, and pulmonary tuberculosis or lung cancer was suspected. The patient was therefore transferred to our hospital for further investigations.

Clinical examination revealed a thin man with wheezing respiration and mild pharyngitis. He was mildly pyrexial at $37.6^{\circ} \mathrm{C}$, and his blood pressure was $95 / 69 \mathrm{~mm} \mathrm{Hg}$. A 24-hour urine collection had a volume of $5860 \mathrm{~mL}$, contained $70.3 \mathrm{~g}$ glucose and yielded a creatinine clearance of $67 \mathrm{~mL} / \mathrm{min}$. 

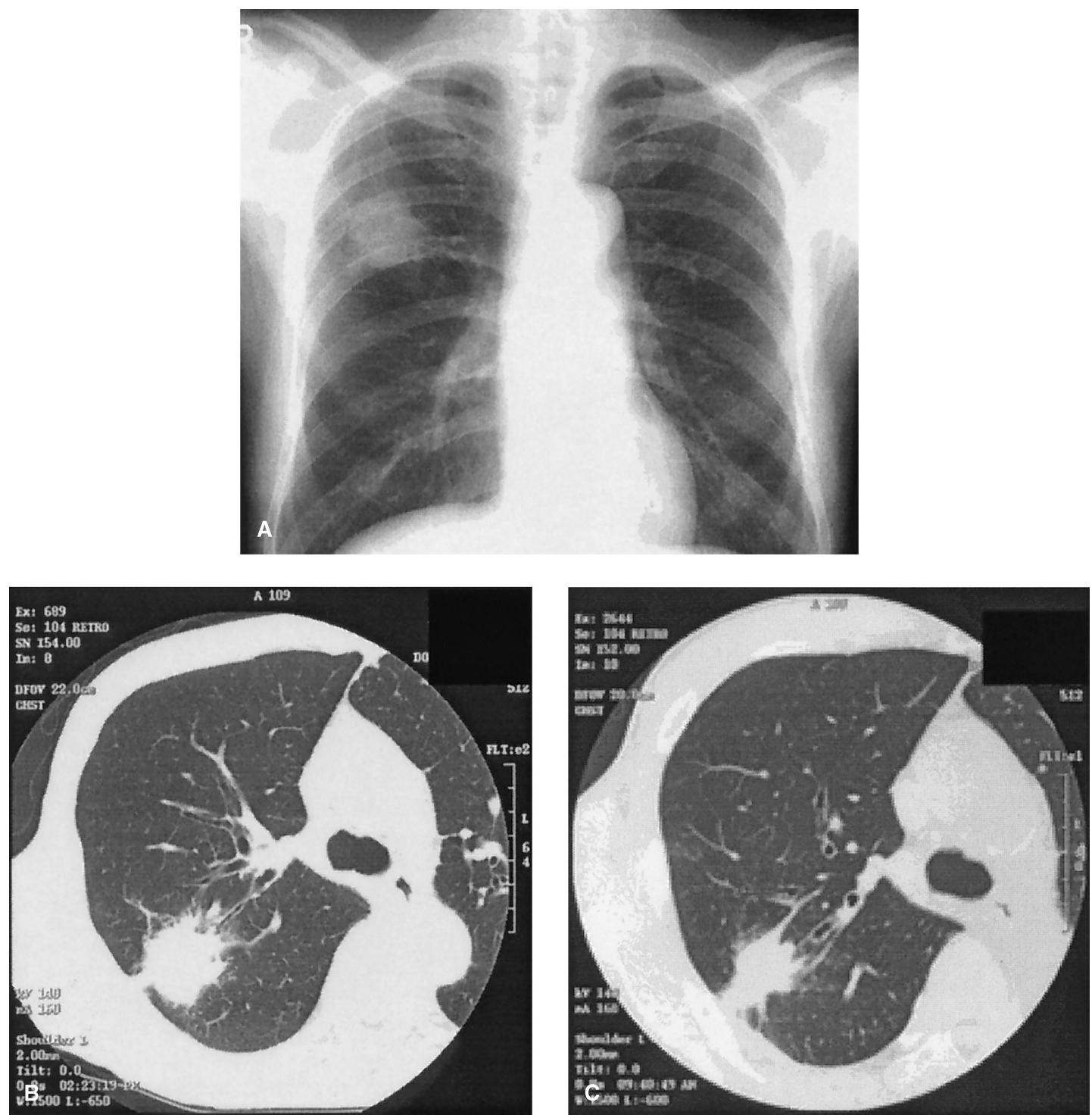

Figure 1. Chest X-ray film (A) and computed tomographic scan (B) on admission, revealing round opacity in right upper lobe. Chest computed tomographic scan (C) taken 6 months after start of therapy, revealing significant reduction in opacity.

Liver function tests revealed total bilirubin of $60 \mathrm{mg} / \mathrm{dL}$, an alkaline phosphatase activity of 342 IU/L, an aspartate aminotransferase activity of $54 \mathrm{IU} / \mathrm{L}$, and a $\gamma$-glutamyl transpeptidase activity of $213 \mathrm{IU} / \mathrm{L}$. The blood urea nitrogen level was $12.9 \mathrm{mg} / \mathrm{dL}$, and the blood glucose level was $301 \mathrm{mg} / \mathrm{dL}$. Hematologic investigations showed hypochromic microcytic anemia with a hematocrit of $33.7 \%$ and a red blood cell count of $3,420,000$ cells $/ \mathrm{mm}^{3}$. The white blood cell count was 3400 cells $/ \mathrm{mm}^{3}$.

Chest radiographs (Figure 1, $A$ ) revealed nodular infiltration in the right midzone. Chest computed tomographic scan (Figure 1, B) demonstrated an opacity in the ventral segment of the upper lobe.

Pulmonary tuberculosis was suspected, but the patient's sputum and bronchoalveolar lavage specimens yielded negative results for acid-fast bacilli on smear and after prolonged culture. A transbronchial lung biopsy was therefore performed in the inferior subsegment of the right upper lobe. Transbronchial lung biopsy tissue sections were stained with hematoxylin and eosin and exhibited an inflammatory cell infiltrate, together with organisms that appeared as broad, nonseptate hyphae (Figure 2). A diagnosis of pulmonary mucormycosis was therefore made.

The patient's general medical and nutritional condition was poor, and it was considered that he could not face a surgical resection. He therefore underwent bronchoscopy in the supine position. After the administration of $5 \%$ lidocaine, the bronchoscope was passed into the right main bronchus to reach the area of infection. Radiographic monitoring of the bronchoscopy 


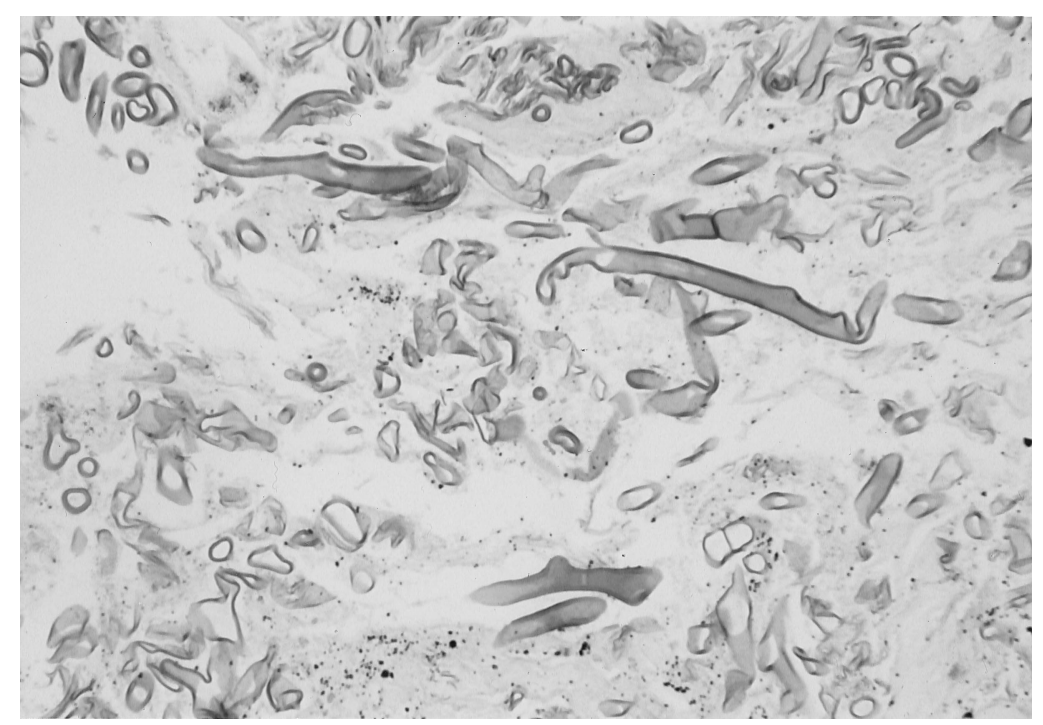

Figure 2. Photomicrograph of transbronchial lung biopsy specimen from right upper lobe. Hematoxylin and eosin stain of biopsy specimen revealed druse of Mucor. Organisms appear as broad, nonseptate hyphae.

was performed to ensure accurate positioning before the instillation of $10 \mathrm{~mL}$ amphotericin B (Fungizone, $2 \mathrm{mg} / \mathrm{mL}$ ) into the lesion. This was repeated seven times during a 1-month period. The patient was also treated with aerosolized amphotericin B (1 $\mathrm{mL}$ volume at $5 \mathrm{mg} / \mathrm{mL}$ administered twice daily by nebulizer) and intravenous amphotericin B (25 $\mathrm{mg}$ on alternate days). Blood sugar levels were controlled with insulin injections.

A repeated chest computed tomographic scan (Figure 1, C) performed 6 months later revealed a significant reduction in opacity at the same level, and the patient was subsequently discharged from the hospital. He continued to receive the aerosolized amphotericin B treatment at his home for 1 year and has made a complete recovery.

\section{Discussion}

This patient had an opacity in his right lung develop during the course of a flu-like prodromal illness. The clinical symptoms and the chest radiographic abnormalities were highly suggestive of pulmonary tuberculosis, but sputum and bronchoalveolar lavage specimens were both smear and culture negative for mycobacteria. Finally, transbronchial lung biopsy revealed a druse of Mucor, a definitive diagnosis of pulmonary mucormycosis was made, and treatment with amphotericin B was initiated.

To our knowledge, this is the first report of curative treatment of pulmonary Mucorales by topical treatment alone. ${ }^{4}$ Mucormycosis is currently a rare disease, but it is likely that patient numbers will increase in the future in parallel with the increases in patients with leukemia, diabetes, dialysis, immunodeficiency syndromes, and transplants. ${ }^{5}$ Immunosuppressed transplant recipients should be followed up carefully so that mycoses may be detected at an early stage, thereby facilitating nonsurgical management, which is the preferred option. Amphotericin B is the antifungal drug of choice and should prove to be effective for mucormycosis. However, amphotericin B toxicity is well described, and the bronchoscopic injection of amphotericin B could be used to treat the lesion directly and thereby avoid potential systemic toxicity. Aerosolized and intravenous amphotericin B preparations were also used successfully as adjunctive therapy in the treatment of this patient. Our results indicate the efficacy of bronchoscopic injection of amphotericin B in the treatment of pulmonary mucormycosis.

\section{References}

1. Nosari A, Oreste P, Montillo M, Carrafiello G, Draisci M, Muti G, et al. Mucormycosis in hematologic malignancies: an emerging fungal infection. Hematologica. 2000;85:1068-71.

2. Kitabayashi A, Hirokawa M, Yamaguchi A, Takatsu H, Miura AB. Invasive pulmonary mucormycosis with rupture of the thoracic aorta. Am J Hematol. 1998;58:326-9.

3. Lehrer RI, Howard DH, Sypherd PS, Edwards JE, Segal GP, Winston DJ. Mucormycosis. Ann Intern Med. 1980;93:93-108.

4. Tedder M, Spratt JA, Anstadt MP, Hegde SS, Tedder SD, Lowe JE. Pulmonary mucormycosis: results of medical and surgical therapy. Ann Thorac Surg. 1994;57:1044-50.

5. Eucker J, Sezer O, Graf B, Possinger K. Mucormycoses. Mycoses. 2000;44:253-60. 